\title{
High Performance Business Computing
}

\author{
Guido Schryen • Natalia Kliewer • Andreas Fink
}

Published online: 11 November 2019

(c) Springer Fachmedien Wiesbaden GmbH, ein Teil von Springer Nature 2019

This special issue addresses High Performance Business Computing (HPBC), which is the application of models, methodologies, tools and technologies of High Performance Computing (HPC) (e.g., Hager and Wellein 2010) to business problems. Its neighbor discipline, High Performance Technical Computing (HPTC), has already found entrance into a variety of scientific disciplines, offering an impressive portfolio of results and further research opportunities. For example, in medicine the cure for Alzheimer's disease may come from a leap forward in HPC as researchers have started surveying an immense number of genomes and assembling these back into pictures, requiring a huge amount of data (680 GB per genome) to be processed (German Center for Neurodegenerative Diseases 2017; Hopkins 2017). In biophysics, scientists have resolved the HIV-1 capsid's chemical structure through parallel molecular dynamics simulations on a supercomputer (Zhao et al. 2013). In earth system sciences, researchers have succeeded in simulating the world-wide weather with extreme high resolution $(870 \mathrm{~m})$ (Yashiro

\footnotetext{
G. Schryen $(\bowtie)$

Paderborn University, Warburger Straße 100, 33098 Paderborn,

Germany

e-mail: guido.schryen@upb.de

URL: http://www.misor.org

\section{N. Kliewer}

Freie Universität Berlin, Garystr. 21, 14195 Berlin, Germany e-mail: natalia.kliewer@fu-berlin.de

URL: http://www.wiwiss.fu-berlin.de/kliewer
}

\author{
A. Fink \\ Institut für Informatik, Helmut-Schmidt-Universität Hamburg, \\ Holstenhofweg 85, 22043 Hamburg, Germany \\ e-mail: andreas.fink@hsu-hamburg.de \\ URL: https://www.hsu-hh.de/ifi/team/fink
}

et al. 2014) and in simulating and visualizing hurricanes for the purpose of forecasting (National Center for Atmospheric Research 2018). In astrophysics, researchers explore the universe with radio telescopes and make use of graphics processing units (GPUs) to process huge volume of data (160 GB/s) (Square Kilometre Array Project 2019).

Acknowledging the advantages and potential that HPC brings to many sciences through technical computing, one might wonder whether HPC also has the potential to address substantial problems occurring in business research and economics. Interestingly, HPC has already contributed to the computational branches of several subfields of business research and economics. For example, in computational economics researchers have started using supercomputers to calculate complex economic problems by approximating high-dimensional functions and solving systems of non-linear equations (e.g., Eftekhari et al. 2017); models of such sizes cannot be calculated using conventional methods. In computational finance, HPC even has a tradition with its history going back to the 1990s (e.g., Zenios 1999; Dempster et al. 2018). An obvious application field of HPC is (big) data analytics and machine learning, where huge amounts of data acquired from sensors, mobile phones, social networks, etc., are analyzed for describing phenomena, predicting developments, and informing strategic decisions. The availability of massive volumes of data, increasing HPC processing capabilities, new machine learning and data mining approaches, and new software frameworks for data processing, such as Hadoop, have led to research activities and avenues for business analytics, social network analysis, global health management (e.g., Wang et al. 2016; Zhang et al. 2016). They further allow moving from offline analytics to realtime analytics. Finally, in the fields of management science and operations research, computing clusters have been used 
to execute parallel algorithms for a broad set of both theoretically and practically relevant problems in scheduling, logistics, and further application fields of optimization and simulation (e.g., Gendron and Crainic 1994; Aldasoro et al. 2017).

While HPC has gained entrance into business research and economics, it is still far away from being an established and appropriately well exploited research approach, despite its large potentials for these fields. The scientific development and practical deployment of HPBC is useful, if not even necessary, for essentially three reasons, which condense the aforementioned arguments:

- The fields of business research and economics become increasingly computational with the advent of large volumes of data and the pervasion of computing resources in business, scientific and social environments. Expectations and business opportunities that result from these developments include the potentials of real-time decision making (e.g., for IT security infrastructure protection and financial stock trading), solving computationally hard optimization problems (e.g., in production and logistics), and analyzing huge volumes of data acquired from sensors, mobile phones, and social networks (e.g., for social network analysis, fraud detection and business analytics).

- Second, HPC infrastructures, tools, libraries, and services are becoming commodity goods offered by computing centers of universities, public cloud providers (e.g., as part of Amazon Web Services, Microsoft Azure, and the Google Cloud Platform), and open source communities (e.g., Apache Hadoop framework). The high relevance of services enabled through HPC is stressed by the recent acquisition of the supercomputer pioneer Cray by Hewlett Packard Enterprise.

- Third, the end of exponential growth in single processor core performance, which has been a key driver of the economic and technology-advancement cycles over the past decades, marks the end of the dominance of the single microprocessor in computing, and the era of sequential computing must give way to a new era in which parallelism is at the forefront (Fuller and Millet 2011). However, sequential programs will not benefit from increases in processor performance that stem from the use of parallel processing capabilities.

In this special issue, one research paper and an interview provide insights along these lines. In the article Efficient Model Points Selection in Insurance by Parallel Global Optimization using Multi CPU and Multi GPU, the authors apply parallel computing to a problem occurring in asset liability management in the insurance sector. In the interview with Utz-Uwe Haus, who is senior researcher at the CRAY European Research Lab, we discuss issues such as the success factors for exploiting the potentials of HPBC, recent technological developments and challenges, as well as business models for making HPBC available to industries.

Acknowledging that a) future enhancements in computing performance will come methodologically primarily from parallelization, b) business research and economics need to exploit computational performance enhancements to address several of its problems more effectively and efficiently, and c) massive parallel computing and HPC capabilities become commodity goods for researchers, we believe and hope that early success stories in HPBC will encourage researchers to step into HPBC and to shape a promising and exciting field of research.

We also welcome further submissions on HPBC in the BISE-Department Decision Analytics and Data Science to follow this Special Issue.

\section{References}

Aldasoro U, Escudero LF, Merino M, Perez G (2017) A parallel branch-and-fix coordination based matheuristic algorithm for solving large sized multistage stochastic mixed $0-1$ problems. Eur J Oper Res 258:590-606

Dempster MAH, Kanniainen J, Keane J, Vynckier E (eds) (2018) High-performance computing in finance: problems, methods, and solutions. CRC Press, Boca Raton

Eftekhari A, Scheidegger S, Schenk O (2017) Parallelized dimensional decomposition for large-scale dynamic stochastic economic models. In: Proceedings of the platform for advanced scientific computing conference, Lugano. ACM, New York, Article 9

Fuller SH, Millet LI (eds) (2011) The future of computing performance: Game over or next level?. National Academies Press, Washington

Gendron B, Crainic TG (1994) Parallel branch-and-bound algorithms: survey and synthesis. Oper Res 42(6):1042-1066

German Center for Neurodegenerative Diseases (2017) Fighting oblivion with a new kind of computer brain. https://www.dzne. de/en/news/public-relations/press-releases/press/fighting-obliv ion-with-a-new-kind-of-computer-brain/. Accessed 28 Oct 2019

Hager G, Wellein G (2010) Introduction to high performance computing for scientists and engineers. CRC Press, Boca Raton

Hopkins C (2017) Systems medicine: the cure for Alzheimer's may come from a leap forward in high-performance computing. Hewlett-Packard Enterprise. https://www.hpe.com/us/en/ insights/articles/systems-medicine-the-cure-for-alzheimers-maycome-from-a-leap-forward-in-high-performance-computing1711.html. Accessed 28 Oct 2019

National Center for Atmospheric Research (2018) Hurricane Odile. https://visgallery.ucar.edu/hurricane-odile/. Accessed 28 Oct 2018

Square Kilometre Array Project (2019) https://www.skatelescope.org/ the-ska-project/. Accessed 28 Oct 2018

Wang Q, Li B, Wang P, Yang J (2016) Using TB-sized data to understand multi-device advertising. In: Proceedings of the international conference on information systems, Dublin. https:// aisel.aisnet.org/icis2016/DataScience/Presentations/13/. Accessed 3 Nov 2019 
Yashiro H, Satoh M, Tomita H (2014) NICAM $870 \mathrm{~m}$ mesh simulations on the $\mathrm{K}$ computer and a road toward global LES. In: American geophysical union fall meeting, San Francisco

Zenios SA (1999) High-performance computing in finance: the last 10 years and the next. Parallel Comput 25(13-14):2149-2175

Zhang K, Bhattacharyya S, Ram S (2016) Large-scale network analysis for online social brand advertising. MIS Q 40(4):849-868
Zhao G, Perilla JR, Yufenyuy EL, Meng X, Chen B, Ning J, Ahn J, Gronenborn AM, Schulten K, Aiken C, Zhang P (2013) Mature HIV-1 capsid structure by cryo-electron microscopy and allatom molecular dynamics. Nature 497:643-646 